Research Paper

\title{
The Traditional Chinese Medicine Baicalein Potently Inhibits Gastric Cancer Cells
}

\author{
Jiasheng $\mathrm{Mu}^{1,2^{*}}$, Tianrun $\mathrm{Liu}^{3^{*}}$, Lin Jiang ${ }^{1,2}$, Xiangsong $\mathrm{Wu}^{1,2}$, Yang $\mathrm{Cao}^{1,2}$, Maolan $\mathrm{Li}^{1,2}, \mathrm{Qian}$ Dong1,2匹 , \\ Yingbin Liu ${ }^{1,2}$, Haineng $\mathrm{Xu}^{4 \bowtie}$ \\ 1. Department of General Surgery, Xinhua Hospital, affiliated to School of Medicine, Shanghai Jiao Tong University, Shanghai 200092, China; \\ 2. Institute of Biliary Tract Disease, Shanghai Jiao Tong University School of Medicine, Shanghai 200092, China; \\ 3. Department of Otorhinolaryngology - Head and Neck Surgery, The Sixth Hospital of Sun Yat-sen University, Guangzhou 510655, China. \\ 4. Department of Radiation Oncology, University of Pennsylvania School of Medicine, Philadelphia, PA 19104, USA. \\ * Jiasheng Mu and Tianrun Liu contributed equally to this work.
}

$\square$ Corresponding author: Dr. Haineng Xu, 3400 Civic Center Blvd., department of Radiation Oncology, University of Pennsylvania School of Medicine, Philadelphia, PA 19104, USA. Email: xuhaineng123@163.com. Or Dr. Yingbin Liu, 1665 Kong-Jiang Road, Department of General Surgery, Xinhua Hospital, Affiliated to School of Medicine, Shanghai Jiao Tong University, Shanghai 200092, China. Email: laoliulyb@sina.com. Or Dr. Qian Dong, 1665 Kong-Jiang Road, Department of General Surgery, Xinhua Hospital, Affiliated to School of Medicine, Shanghai Jiao Tong University, Shanghai 200092, China. Tel and Fax: 86-21-25077885. Email:dongqianqian71@163.com.

() Ivyspring International Publisher. Reproduction is permitted for personal, noncommercial use, provided that the article is in whole, unmodified, and properly cited. See http://ivyspring.com/terms for terms and conditions.

Received: 2015.08.13; Accepted: 2015.12.08; Published: 2016.01.29

\begin{abstract}
Baicalein, a traditional Chinese medicine, is a member of the flavone subclass of flavonoids. It has been reported to have anticancer activities in several human cancer cell lines in vitro. However, the therapeutic effects of baicalein on human gastric cancer and the mechanisms of action of baicalein have not been extensively studied. In the present study, we utilized a cell viability assay and an in vivo tumor growth assay to test the inhibitory effects of baicalein on gastric cancer. Analyses of the cell cycle, apoptosis and alterations in protein levels were performed to elucidate how baicalein functions in gastric cancer. We found that baicalein could potently inhibit gastric cancer cell growth and colony formation. Baicalein robustly induced arrest at the $\mathrm{S}$ phase in the gastric cancer cell line SGC-7901. It induced SGC-7901 cell apoptosis and disrupted the mitochondrial membrane potential $\left(\Delta \Psi_{\mathrm{m}}\right)$ in a dose-dependent manner. Analysis of protein expression levels in SGC-7901 cells showed downregulation of $\mathrm{Bcl}-2$ and upregulation of $\mathrm{Bax}$ in response to baicalein treatment. These results indicate that baicalein induces apoptosis of gastric cancer cells through the mitochondrial pathway. In an in vivo subcutaneous xenograft model, baicalein exhibited excellent tumor inhibitory effects. These results indicate that baicalein may be a potential drug for gastric cancer therapy.
\end{abstract}

Key words: baicalein, traditional Chinese medicine, gastric cancer, $\mathrm{S}$ phase arrest, apoptosis, mitochondria.

\section{Introduction}

Gastric cancer is one of the most aggressive cancers of the digestive tract, and it is the fourth most common cancer with an annual incidence of over 989,000 and mortality of approximately $738,000^{1}$. Surgical resection is the main approach of curative treatment for this highly lethal malignancy. However, due to its non-specific symptoms and highly invasive characteristics, only $30-50 \%$ of patients who underwent surgical exploration can be operated with curative intent surgery. The 5-year survival rates of gastric cancer patients at stage I and stage II are approximately $60 \%$ and $34 \%$, respectively ${ }^{2}$. Even when combined with adjuvant chemotherapy or radiotherapy, patients with advanced stage gastric cancer who underwent surgical treatment still showed poor prognosis, and the 5-year survival rate is only approximately $20 \%-30 \%{ }^{3}$. Therefore, more effective therapies are urgently needed for the treatment of gastric cancer.

Baicalein (5,6,7-trihydroxy-2-phenyl-4H-1-benzo 
pyran-4-one), which is extracted from the roots of Scutellariae radix or Scutellaria baicalensis, is a purified flavonoid with a defined chemical structure. It has been reported that baicalein possesses excellent antioxidant and anti-inflammatory activities ${ }^{4,5}$. Baicalein exhibited anti-tumor effects in several types of cancers by inducing cancer cell apoptosis $6-8$ and suppressing metastasis9-11. Baicalein is genotoxic without producing chromosomal alterations and mutagenesis, which are shown to result in severe side effects in chemotherapy. This effect guarantees baicalein as a safe potential drug. Furthermore, baicalein is very cheap, making it a promising therapeutic for cancer treatment. The inhibitory effect of baicalein has been shown in various types of cancers, such as pancreatic cancer, bladder cancer, lung cancer, hepatoma, breast cancer and skin carcinoma ${ }^{6,9-13}$. However, to the best of our knowledge, there are no previous reports on the effects of baicalein on gastric cancer cells. In this study, we used in vitro and in vivo assays to detect the efficacy of baicalein in treating gastric cancer and to investigate the mechanisms of baicalein. This work may provide new insights into the potential therapeutic role of baicalein in gastric cancer.

\section{Materials and Methods}

\section{Cell lines and culture}

The gastric cancer cell line SGC-7901 was purchased from the Cell Bank of Shanghai Institutes for Biological Sciences, Chinese Academy of Sciences. The gastric cancer cell line MKN45 was obtained from the Japanese Riken Cell Bank (Tsukuba, Japan). The SGC-7901 cells were cultured in high-glucose DMEM (Gibco, USA) supplemented with $10 \%$ fetal bovine serum (Gibco, USA), $100 \mu \mathrm{g} / \mathrm{mL}$ streptomycin and $100 \mathrm{U} / \mathrm{mL}$ penicillin (HyClone, USA). MKN45 cells were cultured in RPMI 1640 with 10\% FBS, 100 $\mu \mathrm{g} / \mathrm{mL}$ streptomycin and $100 \mathrm{U} / \mathrm{mL}$ penicillin. All cells were incubated at $37^{\circ} \mathrm{C}$ under a $5.0 \% \mathrm{CO}_{2}$ atmosphere.

\section{Drug}

Baicalein was purchased from Sigma-Aldrich (St. Louis, USA). It was dissolved in DMSO at 100 $\mathrm{mmol} / \mathrm{L}$ as a stock solution and kept in the dark at $-20^{\circ} \mathrm{C}$. Baicalein was diluted in high-glucose DMEM to treat gastric cancer cells at the indicated dosages.

\section{3-(4, 5-Dimethylthiazol-2-yl)-2, 5- diphenyltetrazolium (MTT) assay}

Drug sensitivity was detected using the MTT assay ${ }^{14}$. Briefly, cells were trypsinized and plated into 96-well plates (Corning, USA) at a density of $5 \times 10^{3}$ cells per well. The cells were cultured overnight and then replenished with fresh medium containing bai- calein at the indicated concentrations. The cells were then incubated for 24,48 or $72 \mathrm{~h}$. A total of $20 \mu \mathrm{L}$ of MTT (Sigma-Aldrich) dissolved in PBS at $5 \mathrm{mg} / \mathrm{mL}$ was added directly to the wells at the indicated times. The plates were then incubated for an additional $4 \mathrm{~h}$ at $37^{\circ} \mathrm{C}$ for the MTT reaction. The supernatant was removed $4 \mathrm{~h}$ later. A total of $100 \mu \mathrm{L}$ of DMSO was added to dissolve the formed formazan crystals, and the optical density was measured at $490 \mathrm{~nm}$ on a microplate reader (Bio-Tek, USA). The results are represented as the average value of 3 independent experiments. The percentage of viable cells was calculated as Cell viability $(\%)=(\mathrm{OD}$ of treatment $/ \mathrm{OD}$ of control) $\times 100$.

\section{Colony formation assay}

SGC-7901 cells were digested into single cell suspension with trypsin-EDTA (Gibco, USA) solution. A total of $2 \mathrm{~mL}$ of the cell suspension was seeded onto 6-well plates (Corning, USA) at a density of 200 cells $/ \mathrm{mL}$. After adherence, the cells were treated with baicalein $(0,15,30$, and $60 \mu \mathrm{mol} / \mathrm{L})$ for $48 \mathrm{~h}$. The supernatant was replaced with fresh medium, and the cells were cultured for an additional 15 days. The cells were then fixed with $10 \%$ formalin and stained with $0.1 \%$ crystal violet (Sigma-Aldrich) for $1 \mathrm{~h}$. Then, the cells were washed and dried. Digital images were taken using a microscope (Leica, Germany). The results represented the average of 3 independent experiments.

\section{Cell cycle analysis}

SGC-7901 cells were treated with baicalein $(0,30$, 60 , and $120 \mu \mathrm{mol} / \mathrm{L})$ for $48 \mathrm{~h}$. The cells were collected and fixed with cold $70 \%$ ethanol at $-20^{\circ} \mathrm{C}$ overnight. Then, the cells were washed and resuspended in cold PBS and incubated at $37^{\circ} \mathrm{C}$ for $30 \mathrm{~min}$ with $10 \mathrm{mg} / \mathrm{mL}$ RNase and $1 \mathrm{mg} / \mathrm{mL}$ propidium iodide (Sigma-Aldrich). DNA content was detected by flow cytometry (BD, San Diego, USA). The percentage of cells at different cell cycle phases was analyzed using the Cell Quest acquisition software (BD Biosciences).

\section{Flow cytometric analysis of cell apoptosis}

The Annexin V/propidium iodide (PI) assay was performed according to the manufacturer's instructions (Invitrogen, USA). Briefly, SGC-7901 cells were plated into 6-well plates (Corning, USA) and incubated for $48 \mathrm{~h}$ with baicalein $(0,15,30$, and 60 $\mu \mathrm{mol} / \mathrm{L})$. The cells were collected, washed with cold PBS, centrifuged and resuspended in $100 \mu \mathrm{l}$ binding buffer containing $2.5 \mu \mathrm{l}$ FITC-conjugated Annexin V and $1 \mu \mathrm{ll} 100 \mu \mathrm{g} / \mathrm{ml}$ PI. The cells were incubated for 15 $\mathrm{min}$ at room temperature in the dark. A total of 10000 events were collected by flow cytometry (BD, San Diego, USA). 


\section{Detection of morphological apoptosis with Hoechst 33342 staining}

After being treated with baicalein $(0,15,30$, and $60 \mu \mathrm{mol} / \mathrm{L})$ for $48 \mathrm{~h}$, the SGC-7901 cells were washed with PBS and fixed with methanol:acetic acid (3:1) for $15 \mathrm{~min}$ at room temperature. The cells were washed with PBS and stained with $5 \mu \mathrm{g} / \mathrm{mL}$ Hoechst 33342 for $10 \mathrm{~min}$. Alterations in morphology of the nuclei were observed using fluorescence microscopy (Leica, Germany).

\section{Detection of mitochondrial membrane poten- tial $(\Delta \Psi \mathrm{m})$ variation with fluorescence $\mathrm{mi}-$ croscopy}

The $\Delta \Psi \mathrm{m}$ was analyzed by fluorescence microscopy using the 5,50,6,60-tetrachloro-1,10,3,30-tetraethylbenzimidazol carbocyanine iodide (JC-1) probe. The SGC-7901 cells were treated with baicalein $(15,30$, and $60 \mu \mathrm{mol} / \mathrm{L})$ for $48 \mathrm{~h}$. A total of $5 \mu \mathrm{l} \mathrm{JC}-1$ staining solution (Beyotime, China) was added to cells, and the cells were incubated in a $5 \% \mathrm{CO} 2$ atmosphere at $37^{\circ} \mathrm{C}$ for $20 \mathrm{~min}$ in the dark. After washing twice with buffer, SGC-7901 cells were observed under fluorescence microscopy (Leica, Germany).

\section{Western blot analysis}

Western blotting was performed as previously described ${ }^{16}$. SGC-7901 cells were treated with various concentrations of baicalein $(0,15,30,60$, and 120 $\mu \mathrm{mol} / \mathrm{L})$ for $48 \mathrm{~h}$ and then lysed in sample buffer, followed by denaturation. The protein concentration was measured using the bicinchoninic acid assay system (Beyotime, China) with BSA as a standard protein. For each sample, $80 \mu \mathrm{g}$ total protein was separated by SDS-PAGE gel and electrophoretically transferred to nitrocellulose membranes. The membranes were blocked with 5\% non-fat milk for $40 \mathrm{~min}$ and incubated with anti-procaspase-3, anti-Bcl-2, anti-Bax, or anti-PARP antibodies (1:1000; Cell Signaling Technology, Danvers, MA) at $4^{\circ} \mathrm{C}$ overnight. The membranes were then incubated with goat anti-rabbit/anti-mouse secondary antibody conjugated with horseradish peroxidase (1:5000; Abcam) and then exposed using films. For proteins with different molecular weights, the PVDF membranes were cut according to the protein marker and then incubated with antibodies. For proteins of similar sizes, the membranes were stripped and then incubated with other antibodies, including $\beta$-actin antibody as an internal control, then the same steps were performed as described above. Films were scanned, and the optical intensity of the procaspase-3, Bcl-2, Bax, PARP, and $\beta$-actin bands were quantified with Quantity One. $\beta$-actin was used as the internal control. The ratios of procaspase-3/actin, Bcl-2/actin, Bax/actin, and $\mathrm{PARP} /$ actin were calculated in each group, and these represent the transcriptional levels of the respective proteins.

\section{Animal experiments}

All of the animal experiments were approved by the Animal Care and Use Committee of Shanghai Jiao Tong University. The experiments were performed according to the U.S. Public Health Service Policy on Humane Care and the Use of Laboratory Animals. Five-week-old male BALB/c nude mice were purchased from the Shanghai Laboratory Animal Center (SLAC; Shanghai, China). The mice were subcutaneously injected with $1 \times 10^{6}$ SGC-7901 cells. After the tumors were visible, either vehicle, low dose baicalein $(15 \mathrm{mg} / \mathrm{kg})$ or high dose baicalein $(50 \mathrm{mg} / \mathrm{kg})$ was administered to the mice intragastrically for 1 week. The drug was given daily for a total of 7 treatments. After treatment, the size of the tumors was measured every week and was calculated as length $\times$ width $\times$ width/2. The mice were sacrificed 4 weeks post-treatment. The tumors were isolated, and pictures were taken.

\section{Statistical analysis}

All of the values are expressed as the mean \pm SD and analyzed by Student's $t$-test using SPSS version 13.0 software. A $p$-value less than 0.05 was considered significant.

\section{Results}

\section{Baicalein suppressed the growth of gastric cancer cells}

To examine the biological effect of baicalein (Fig 1A), SGC-7901 and MKN45, cells were treated with baicalein for the indicated times, and cell viability was measured. Baicalein decreased the proliferation of SGC-7901 cells in a dose- and time-dependent manner (Fig 1B \& Supplementary Figure 1).

To test the long-term effect of baicalein on cell growth, SGC-7901 cells were treated with 0, 15, 30, and $60 \mu \mathrm{mol} / \mathrm{L}$ baicalein for $48 \mathrm{~h}$ and cultured for another 15 days. High dose baicalein decreased the number of colonies, the diameter of colonies and the area of colonies (Fig 1C \& 1D). Colony formation was robustly suppressed by baicalein at $30 \mu \mathrm{mol} / \mathrm{L}$. These results demonstrated that baicalein exhibits potent anti-proliferation effects in gastric cancer cells.

\section{Baicalein induces gastric cancer cells $\mathbf{S}$ phase arrest}

To gain insights into the mechanism by which baicalein inhibited gastric cancer growth, we measured the cell cycle distribution of SGC-7901 cells with 
baicalein treatment. In comparison to DMSO-treated cells, baicalein induced a significant increase in $S$ phase cells and decreased the percentage of G1 phase cells in a dose-dependent manner. The $S$ phase frac- tion increased from $33.4 \pm 2.6 \%$ to $65.3 \pm 3.1 \%$ in SGC7901 cells after treatment with 0, 30, 60, 120 $\mu \mathrm{mol} / \mathrm{L}$ of baicalein, respectively (Fig 2).
A<smiles>O=c1cc(-c2ccccc2)oc2cc(O)c(O)c(O)c12</smiles>

B

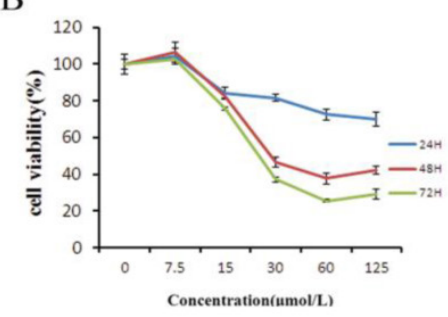

$\mathrm{C}$

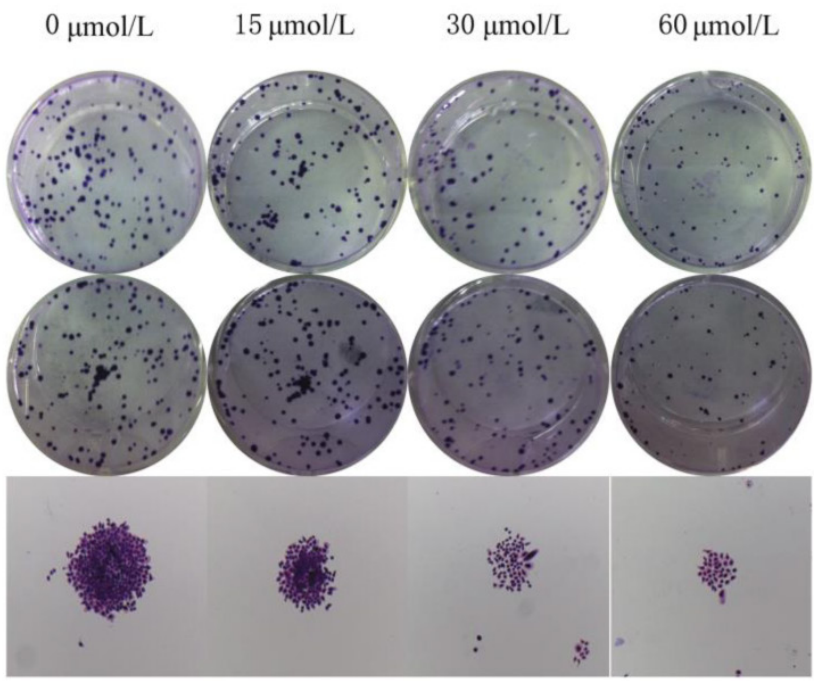

$\mathrm{D}$
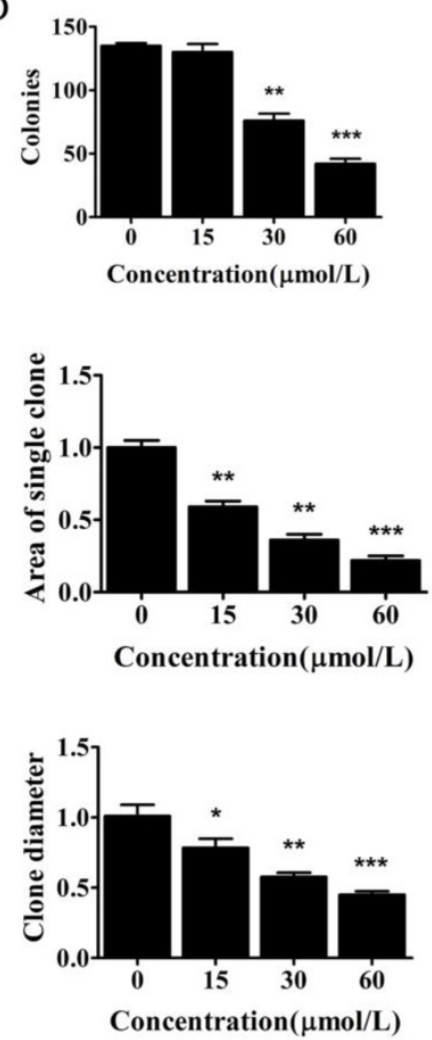

Figure 1. Baicalein suppressed the growth of gastric cancer cells. (A) Chemical structure of baicalein. (B) Assessment of the cell viability of SGC-7901 cells following treatment with baicalein at the indicated concentrations. (C) Detection of colony formation of SGC-7901 cells after treatment with baicalein at the indicated concentrations. (D) Quantification of colony numbers, diameters and areas after treatment with baicalein.

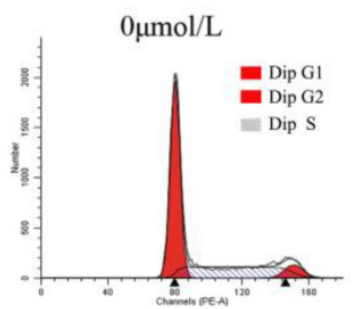

$60 \mu \mathrm{mol} / \mathrm{L}$

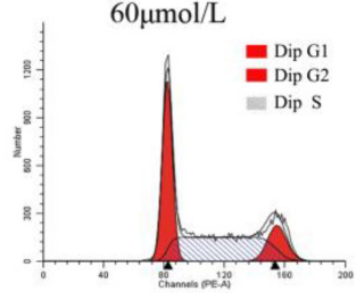

$30 \mu \mathrm{mol} / \mathrm{L}$

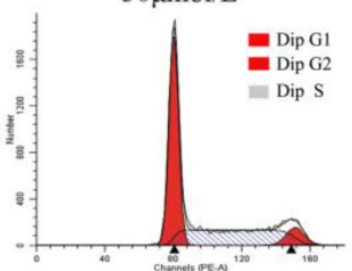

$120 \mu \mathrm{mol} / \mathrm{L}$

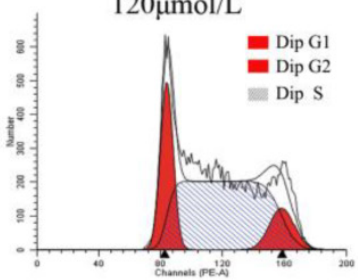

B

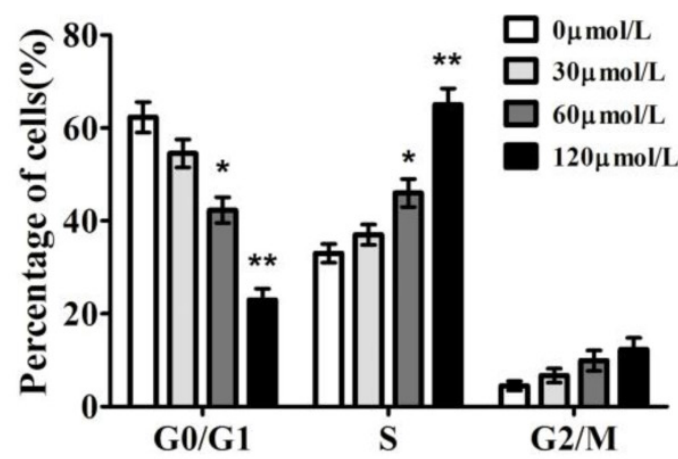

Figure 2. Baicalein induces S phase arrest of gastric cancer cells. (A) Cell cycle detection of SGC-7901 cells following treatment with baicalein at the indicated concentrations. (B) Quantification of G0/G1, S, and G2/M phase cells in baicalein-treated SGC-7901 cells. 


\section{Baicalein induces gastric cancer cell apoptosis}

To test whether baicalein could induce gastric cancer cell death, we used Hoechst staining to observe the morphological alterations of nuclei. In the untreated SGC7901 cells, the observed nuclei were a weak homogeneous blue, whereas bright chromatin condensation and nuclear fragmentation were observed in the group treated with baicalein. The number of apoptotic nuclei containing condensed chromatin increased significantly (Fig 3A), suggesting that baicalein may induce gastric cancer cell apoptosis. To further confirm this, we detected apoptotic cells among the SGC-7901 cells treated with baicalein. Baicalein potently induced SGC-7901 cell apoptosis in a dose-dependent manner (Fig 3B).

A
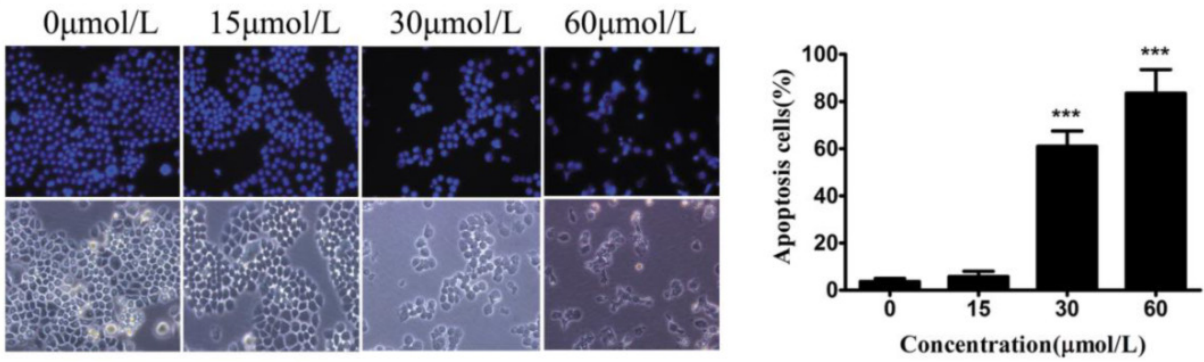

$\mathrm{B}$

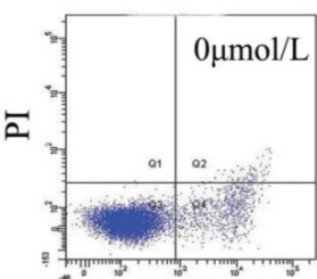

Annexin V

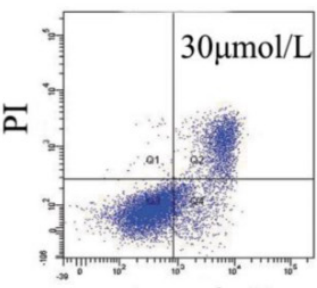

Annexin V

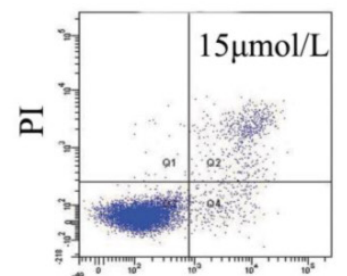

Annexin V

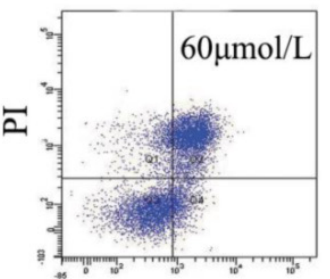

Annexin V

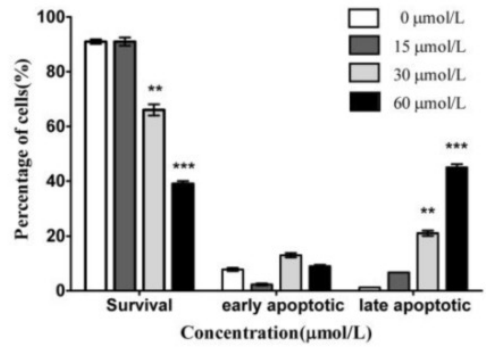

Figure 3. Baicalein induces gastric cancer cell apoptosis. (A) Nuclei staining with Hoechst 33342 of baicalein-treated SGC-7901 cells. Left upper: Hoechst staining. Left lower: Morphology. Right: Quantification of fragmented nuclei. (B) Detection of apoptosis by Annexin V-FITC/PI staining. Right: Quantification of apoptotic cells.
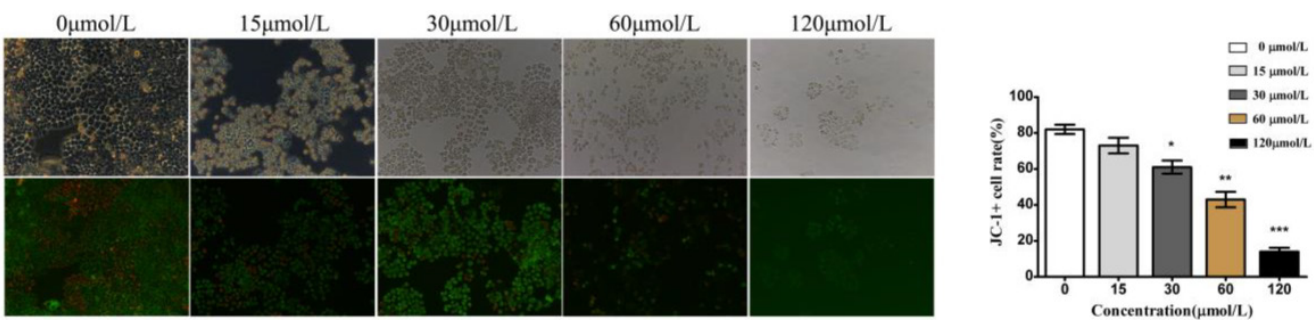

Figure 4. Baicalein decreased the mitochondrial membrane potential $\left(\Delta \Psi_{\mathrm{m}}\right)$ in gastric cancer cells. Left upper: Morphology. Left lower: JC-1 staining. Right: Quantification of cells without disruption of the mitochondria. 


\section{Baicalein regulates $\mathrm{Bcl}-2$ and $\mathrm{Bax}$ expression}

To further elucidate how baicalein induced gastric cancer cell apoptosis, we measured the expression of apoptosis-associated genes. We observed decreased levels of pro-caspase- 3 and increased levels of cleaved PARP, demonstrating the induction of apoptosis by baicalein in gastric cancer cells (Fig 5). Interestingly, we found that baicalein decreased the anti-apoptotic protein Bcl-2 and increased the pro-apoptotic protein Bax (Fig 5). Considering that these two proteins are closely associated with mitochondria, it is possible that baicalein induced apoptosis through mitochondrial disruption by changing the levels of the apoptosis-associated proteins Bcl-2 and Bax.

\section{Baicalein potently inhibited gastric cancer growth in vivo}

To determine whether baicalein could inhibit gastric cancer in vivo, we established a subcutaneous xenograft in nude mice. Baicalein inhibited xenograft growth. Tumors of smaller size were observed after mice were treated with high dose baicalein (Fig 6A). Additionally, the tumor weights of the treated groups were quite smaller than those of control mice (Fig 6B). These results revealed that baicalein could inhibit gastric cancer in vivo.

\section{Discussion}

In recent years, various studies have demonstrated that baicalein, a bioactive flavonoid constituent of Scutellariae radix, is capable of inhibiting tumor cell growth, inducing cell cycle arrest and inducing apoptosis in human hepatocellular carcinoma and lung cancer cell lines $13,17,18$. Doses of $15-50 \mu \mathrm{M}$ baicalein could induce pancreatic cancer cell apoptosis through downregulation of the anti-apoptotic protein Mcl-18. In human bladder cancer cells, $60-80 \mu \mathrm{M}$ baicalein could retard cell growth by inhibiting CDC2 kinase activity ${ }^{6}$. Recent studies also showed that baicalein exhibited anti-proliferative activity in HCC and lung cancer cells ${ }^{12,13}$. Baicalein inhibited cell migration and invasion by inhibiting MMP-2/9 activity in human hepatoma cells ${ }^{9}$ and human breast cancer cells ${ }^{10}$. Moreover, baicalein inhibited cell invasion by inhibiting expression of the anchor protein Ezrin in human skin carcinoma ${ }^{11}$. However, to date, no detailed studies have tested the effects of baicalein on human gastric cancer cells. In this study, we investigated the anti-cancer effects of baicalein in SGC7901, a classic gastric cell line. We also investigated the mechanisms of baicalein in inhibiting gastric cancer cells. To the best of our knowledge, this is the first study of the function and mechanism of action of baicalein in gastric cancer.

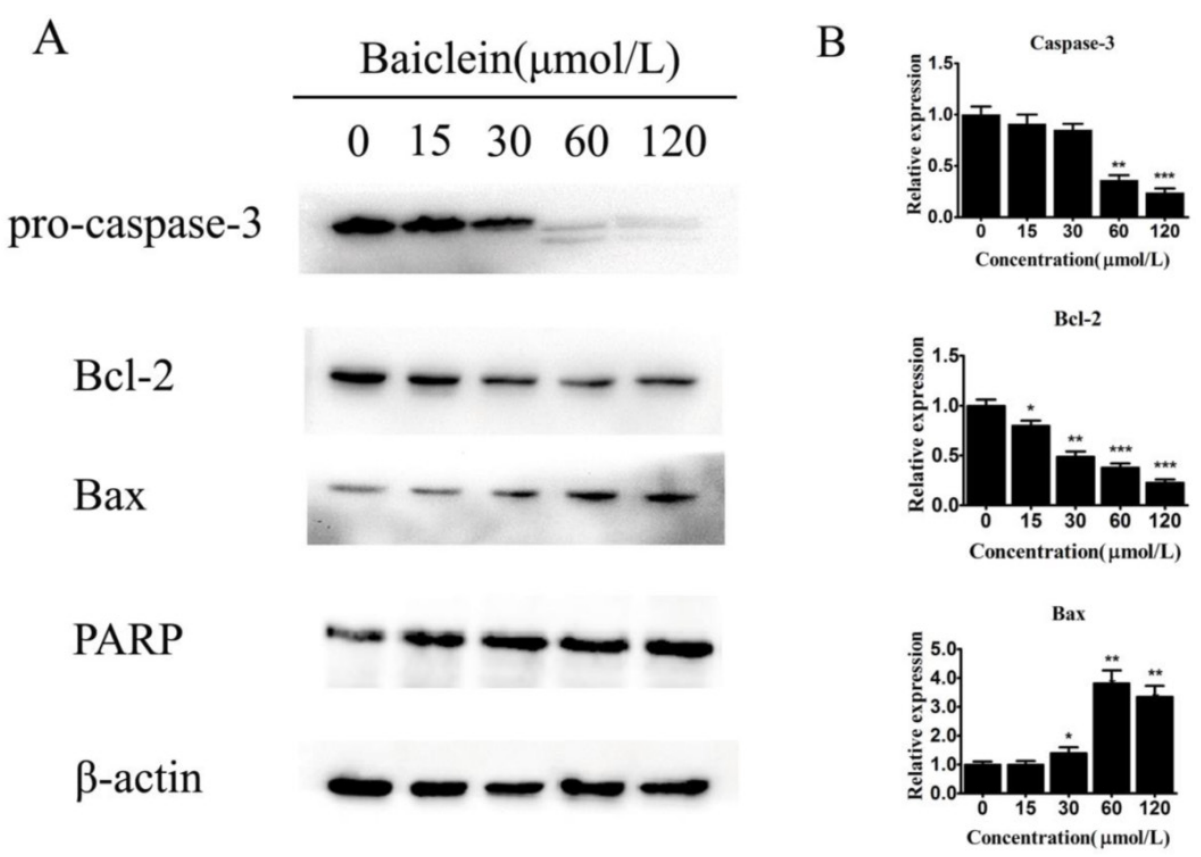

Figure 5. Baicalein regulates Bcl-2 and Bax expression. (A) Expression of pro-caspase-3, Bcl-2, Bax, and cleaved PARP in SGC-7901 cells treated with baicalein at the indicated concentrations. GAPDH was used as an internal control. (B) Quantification of protein expression relative to the internal control GAPDH. 

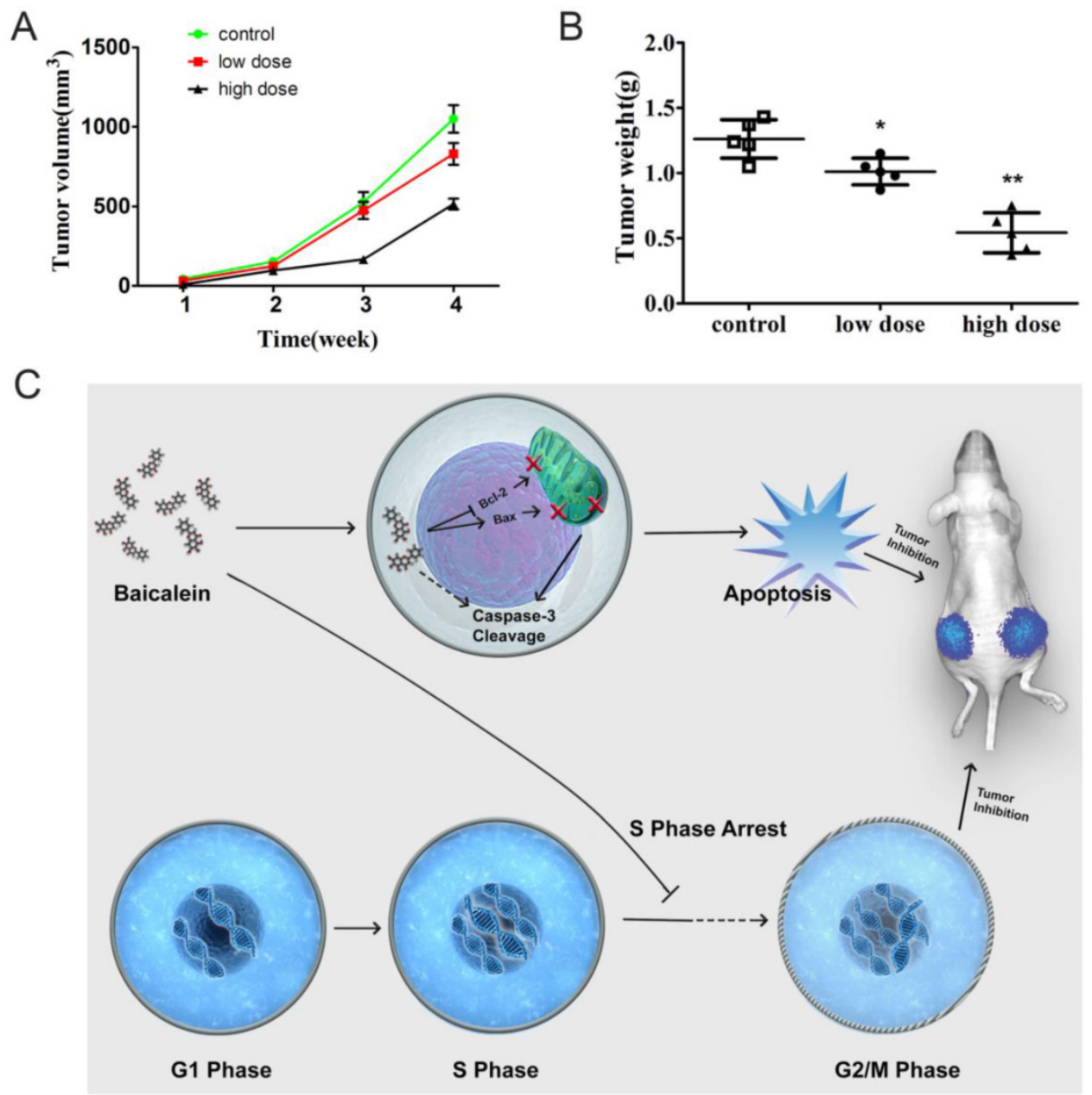

Figure 6. Baicalein potently inhibited gastric cancer growth in vivo. (A) Tumor size of mice treated with baicalein or not at 4 weeks. (B) Tumor weight of mice treated with baicalein or not at 4 weeks. (C) Model of baicalein induced tumor inhibition.

We assessed the anti-tumor activity of baicalein on gastric cancer cells using an MTT assay and a colony forming assay. The results showed that baicalein inhibited cell growth in a dose-dependent manner (Fig 1). Pretreatment with a low dose of baicalein inhibited colony formation of the cells (Fig 1). After the medium was refreshed without drugs, the cells that been treated with baicalein recovered several days later and gradually formed colonies, and this was dependent on the initial dosage of the drug. Because the cells treated with a low dose of baicalein could recover, the possible resistance of cells to baicalein was indicated. This observation needs to be further confirmed in future studies in which cells are treated with low and high dosages of baicalein and their resistance to the drug is tested. Combination with chemotherapy drugs, small molecules or other types of treatment may help to overcome possible resistance. Flow cytometric analysis revealed that baicalein induced cell cycle arrest at the $S$ phase in a dose-dependent manner (Fig 2). Flow cytometric estimation showed that baicalein produced a dose-dependent increase in the apoptotic cell population, suggesting the sequential events of cell cycle arrest followed by apoptosis (Fig 3). Apoptosis was also confirmed by characteristic morphological changes. The morphological changes of apoptosis include membrane blebbing, cell shrinkage, chromatin condensation, and the formation of apoptotic bodies. Staining with Hoechst 33342 clearly revealed condensed, fragmented nuclei and apoptotic bodies in the baicalein-treated cells (Fig 3).

Mitochondria are increasingly recognized as bioenergetics and metabolic centers essential for both life and death ${ }^{19,20}$. Our study revealed a dose-dependent depolarization of the mitochondrial membrane potential, shown by a shift in fluorescence from red to green in baicalein-treated cells. This confirmed that baicalein induces a disturbance of the mitochondrial transmembrane potential, thereby inducing the apoptotic signal through the mitochondrial pathway (Fig 4).

Stimuli could initiate a cascade of molecular events and ultimately lead to the activation of the 
proteolytic enzymes (caspases) responsible for apoptosis ${ }^{21,22}$. However, most cancer cells can block apoptosis, which allows them to survive under the condition of genetic and morphological transformations. Thus, understanding the mechanisms of apoptosis is critical for the prevention and treatment of various types of disorders, including cancers. In general, apoptosis is mediated through two major pathways, the extrinsic (death receptor-mediated) and intrinsic (mitochondrial-mediated) pathways ${ }^{23,24}$. Bcl-2 (B-cell lymphoma 2), encoded by the BCL2 gene in humans, is the founding member of the Bcl-2 family of proteins that regulate apoptosis. Members of this protein family either promote apoptosis or prevent apoptosis ${ }^{25-27}$. Bax (Bcl-2-associated $X$ protein) is an apoptosis regulator belonging to the Bcl-2 gene family. Bax promotes apoptosis by binding to and antagonizing the Bcl-2 protein ${ }^{28}$. Our study showed that baicalein downregulated the expression of $\mathrm{Bcl}-2$ and upregulated the expression of Bax, leading to the induction of cell apoptosis (Fig 5).

We also measured caspase activation and PARP cleavage. Caspases are a family of cysteine proteases that cleave target proteins at specific aspartate residues, and they constitute key components of the apoptotic pathway. Caspase- 3 is a key effector molecule in the caspase-dependent cell apoptosis pathway that cleaves a number of cellular proteins. Thus, activation of the enzymatic activity of caspases-3 might represent a mechanism for initiating the apoptotic process $^{29}$. In the present study, baicalein treatment resulted in the cleavage of PARP and subsequent DNA degradation through the activation of caspase-3 (Fig 5). Two distinct pathways of apoptosis, mitochondria-initiated apoptosis and death receptor-mediated apoptosis, both induced caspase- 3 activation. ${ }^{30}$ Further studies are required to explore the upstream mechanisms. Furthermore, we found that baicalein potently inhibited tumor growth in nude mice (Fig 6). In our experiments, we only administered baicalein intragastrically for 7 days, and this dramatically inhibited the growth of tumors in vivo. A better inhibitory effect of baicalein may be observed after continuous drug treatment.

In our study, we found that baicalein could inhibit gastric cancer cell growth in vitro and in vivo. Baicalein could upregulate the pro-apoptotic gene Bax expression and decreased the anti-apoptotic gene Bcl-2 expression. It disrupted the membrane potential of mitochondrion and led to the cleavage of caspase 3 . The gastric cancer cells underwent apoptosis after baicalein treatment. Moreover, baicalein could also arrest the gastric cancer cells at $S$ phase. The dual-function of baicalein led to the dramatically inhibitory effect on gastric cancer cells in vitro and pre- vented gastric tumors in vivo (Fig 6C). Our study revealed the ability of baicalein to inhibit gastric cancer in vitro and in vivo. These results suggest that baicalein may represent an effective drug for use in gastric cancer therapy.

\section{Supplementary Material}

Supplementary Figure 1.

http://www.jcancer.org/v07p0453s1.pdf

\section{Acknowledgments}

This study was supported by the Foundation of Shanghai Health and Family Planning Commission (No. 20134409), the National Natural Science Foundation of China (No. 81172026, 81272402, and 81172029), the Foundation of Shanghai Outstanding Academic Leaders (No. 11XD1403800), the National High Technology Research and Development Program (863 Program) (No. 2012AA022606), the Post-doctoral Research Foundation of China (No. 2012M511107), the Foundation for Interdisciplinary Research of Shanghai Jiaotong University (No. YG2011ZD07), the Shanghai Science and Technology Commission Inter-governmental International Cooperation Project (No. 12410705900), and the Post-doctoral Research Program of Shanghai (No. 12R21415300).

\section{Competing interests}

The authors have declared that no competing interest exists.

\section{References}

1. Ferlay J, Shin HR, Bray F, Forman D, Mathers C, Parkin DM. Estimates of worldwide burden of cancer in 2008: GLOBOCAN 2008. Int J Cancer. 2010;127(12):2893-2917.

2. Parkin DM, Pisani P, Ferlay J. Global cancer statistics. CA Cancer J Clin. 1999;49(1):33-64, 31.

3. Crew KD, Neugut AI. Epidemiology of upper gastrointestinal malignancies. Semin Oncol. 2004;31(4):450-464

4. Lin CC, Shieh DE. The anti-inflammatory activity of Scutellaria rivularis extracts and its active components, baicalin, baicalein and wogonin. Am J Chin Med. 1996;24(1):31-36.

5. Shao ZH, Vanden Hoek TL, Qin Y, et al. Baicalein attenuates oxidant stress in cardiomyocytes. Am J Physiol Heart Circ Physiol. 2002;282(3):H999-H1006.

6. Chao JI, Su WC, Liu HF. Baicalein induces cancer cell death and proliferation retardation by the inhibition of CDC2 kinase and survivin associated with opposite role of p38 mitogen-activated protein kinase and AKT. Mol Cancer Ther. 2007;6(11):3039-3048.

7. Jiang RH, Su WC, Liu HF, Huang HS, Chao JI. Opposite expression of securin and gamma-H2AX regulates baicalein-induced cancer cell death. J Cell Biochem. 2010;111(2):274-283.

8. Takahashi H, Chen MC, Pham H, et al. Baicalein, a component of Scutellaria baicalensis, induces apoptosis by Mcl-1 down-regulation in human pancreatic cancer cells. Biochim Biophys Acta. 2011;1813(8):1465-1474.

9. Chiu YW, Lin TH, Huang WS, et al. Baicalein inhibits the migration and invasive properties of human hepatoma cells. Toxicol Appl Pharmacol. 2011;255(3):316-326.

10. Wang L, Ling $\mathrm{Y}$, Chen $\mathrm{Y}$, et al. Flavonoid baicalein suppresses adhesion, migration and invasion of MDA-MB-231 human breast cancer cells. Cancer Lett. 2010;297(1):42-48.

11. Wu B, Li J, Huang D, et al. Baicalein mediates inhibition of migration and invasiveness of skin carcinoma through Ezrin in A431 cells. BMC Cancer. 2011;11:527.

12. Du G, Han G, Zhang S, et al. Baicalin suppresses lung carcinoma and lung metastasis by SOD mimic and HIF-1alpha inhibition. Eur J Pharmacol. 2010;630(1-3):121-130. 
13. Chen $\mathrm{CH}$, Huang LL, Huang CC, Lin CC, Lee $\mathrm{Y}$, Lu FJ. Baicalein, a novel apoptotic agent for hepatoma cell lines: a potential medicine for hepatoma. Nutr Cancer. 2000;38(2):287-295.

14. Plumb JA. Cell sensitivity assays: the MTT assay. Methods Mol Med. 2004;88:165-169.

15. Dong $\mathrm{P}$, Zhang $\mathrm{Y}, \mathrm{Gu}$ J, et al. Wogonin, an active ingredient of Chinese herb medicine Scutellaria baicalensis, inhibits the mobility and invasion of human gallbladder carcinoma GBC-SD cells by inducing the expression of maspin. J Ethnopharmacol. 2011;137(3):1373-1380.

16. Quan Z, Gu J, Dong P, et al. Reactive oxygen species-mediated endoplasmic reticulum stress and mitochondrial dysfunction contribute to cirsimaritin-induced apoptosis in human gallbladder carcinoma GBC-SD cells. Cancer Lett. 2010;295(2):252-259.

17. Leung HW, Yang WH, Lai MY, Lin CJ, Lee HZ. Inhibition of 12-lipoxygenase during baicalein-induced human lung nonsmall carcinoma H460 cell apoptosis. Food Chem Toxicol. 2007;45(3):403-411.

18. Chang $\mathrm{WH}$, Chen $\mathrm{CH}$, Gau RJ, et al. Effect of baicalein on apoptosis of the human Hep G2 cell line was induced by mitochondrial dysfunction. Planta Med. 2002;68(4):302-306.

19. Brenner C, Kroemer G. Apoptosis. Mitochondria--the death signal integrators. Science. 2000;289(5482):1150-1151.

20. Degli Esposti M. Mitochondria in apoptosis: past, present and future. Biochem Soc Trans. Jun 2004;32(Pt3):493-495.

21. Earnshaw WC, Martins LM, Kaufmann SH. Mammalian caspases: structure, activation, substrates, and functions during apoptosis. Annu Rev Biochem. 1999;68:383-424.

22. Hengartner MO. The biochemistry of apoptosis. Nature. 2000;407(6805):770-776

23. Yi $\mathrm{CH}$, Yuan J. The Jekyll and Hyde functions of caspases. Dev Cell. 2009;16(1):21-34.

24. Parsons MJ, Green DR. Mitochondria in cell death. Essays Biochem. 2010;47:99-114.

25. Murray A. Cyclin ubiquitination: the destructive end of mitosis. Cell. 1995;81(2):149-152.

26. Antonsson B, Martinou JC. The Bcl-2 protein family. Exp Cell Res. 2000;256(1):50-57.

27. Reed JC. Double identity for proteins of the Bcl-2 family. Nature. 1997:387(6635):773-776.

28. Oltvai ZN, Milliman CL, Korsmeyer SJ. Bcl-2 heterodimerizes in vivo with a conserved homolog, Bax, that accelerates programmed cell death. Cell. 1993;74(4):609-619.

29. Potokar M, Milisav I, Kreft M, Stenovec M, Zorec R. Apoptosis triggered redistribution of caspase-9 from cytoplasm to mitochondria. FEBS Lett. 2003;544(1-3):153-159.

30. Mao WP, Ye JL, Guan ZB, et al. Cadmium induces apoptosis in human embryonic kidney (HEK) 293 cells by caspase-dependent and -independent pathways acting on mitochondria. Toxicol In Vitro. 2007;21(3):343-354. 